\title{
Modeling Wireless Shadow Networks
}

\author{
Filip De Turck \\ Department of Information Technology (INTEC) \\ Ghent University \\ Sint-Pietersnieuwstraat 41 \\ B-9000 Gent, Belgium. \\ filip.deturck@intec.ugent.be
}

\author{
Aurel A. Lazar \\ Department Of Electrical Engineering \\ Columbia University \\ 801 Schapiro Research Building (CEPSR) \\ 530 West 120 th Street, New York, NY \\ 10027-6699, USA. \\ aurel@ee.columbia.edu
}

\begin{abstract}
In case of natural disasters or intentional attacks, telecommunication networks often get heavily damaged and current resilience schemes have proven to be insufficient for rapid recovery of telecommunication services from catastrophic failures. We consider wireless recovery networks as a strong candidate to provide emergency communication resources in case of such severe network outages, and to preserve, with minimal degradation in quality, the distribution of standard communication services.

The focus of our work is on the modeling of wireless shadow networks and study their throughput as a function of the system parameters. We address the design problem of determining the required number of antennas and their most appropriate location for a simple topology. For directional antennas we investigate the expected throughput gain as a function of the beam-width. Furthermore, some interesting topics such as the evaluation of multi-hop constellations and modeling the antenna steering strategies, will be briefly introduced.
\end{abstract}

Categories and Subject Descriptors: C.2 [Computercommunication networks]: Network architecture and design -wireless communication, network communications.

General Terms: Design, Performance, Theory.

Keywords: Wireless recovery networks, Ad hoc networking, Throughput estimation, Node location, Directional antennas.

\section{INTRODUCTION}

\subsection{Motivation}

Resilience studies of telecommunication networks mainly focus on protection and restoration strategies for rapidly recovering from cable cuts or network element failures, that are due to aging or software crashes. In case of protection schemes, each affected connection is switched over to a

Permission to make digital or hard copies of all or part of this work for personal or classroom use is granted without fee provided that copies are not made or distributed for profit or commercial advantage and that copies bear this notice and the full citation on the first page. To copy otherwise, to republish, to post on servers or to redistribute to lists, requires prior specific permission and/or a fee.

MSWiM'04, October 4-6, 2004, Venezia, Italy.

Copyright 2004 ACM 1-58113-953-5/04/0010 ...\$5.00. dedicated backup connection, whereas restoration schemes imply that affected connections are restored at failure detection time. Network dimensioning algorithms, that calculate the required capacity of the network in order to guarantee resilience against single or multiple uncorrelated link or node failures, have been extensively studied for both protection and restoration schemes $([1,2])$.

However, in the case of a major network outage (due to a natural disaster or an intentional attack), protection and restoration schemes are clearly ineffective. For example, during 9/11 the network infrastructure and switching facilities of one of the main network providers located adjacent to the World Trade Center (WTC) towers were largely destroyed. As a result, millions of regular phone lines and mobile phones in the area surrounding the WTC in New York City (NYC) became unusable and there was no network connectivity in parts of the financial district for several days or weeks afterwards.

In order to rapidly provide emergency communication resources, wireless ad hoc networks have been studied in great detail before. Wireless ad hoc networks allow to establish network connectivity in areas with no dedicated infrastructure. The design problem of wireless recovery networks has been subject of research in the military context. However, the usage of wireless recovery networks to largely preserve the distribution of standard communication services in case of catastrophic failures, has not been dealt with before. Installation or activation of reconfigured wireless facilities for restoring the network connectivity is a strong candidate for quickly providing essential communications capabilities. For instance, a wireless recovery network for the $9 / 11$ outage scenario is depicted in Figure 1: antennas installed or activated in the disaster area (financial district of NYC) pick up communication signals from the affected devices and transmit the aggregated signals to antennas in the surrounding areas. The latter antennas ensure the delivery of the received traffic to a wired network that routes the packets to their destination. In order to avoid interference of wireless communication channels inside the affected area, antennas are reconfigured to pick up signals from mobile users or companies' uplinks at a certain frequency and transmit them to the outside antennas at another frequency. Should the distance between the antennas inside or outside the disaster area exceed their coverage range, relay antennas can be placed or activated in between. Thus, such a wireless network can be regarded as a shadow network, endowed with the ability to substitute parts of the wired network with wireless facilities. Recovery 


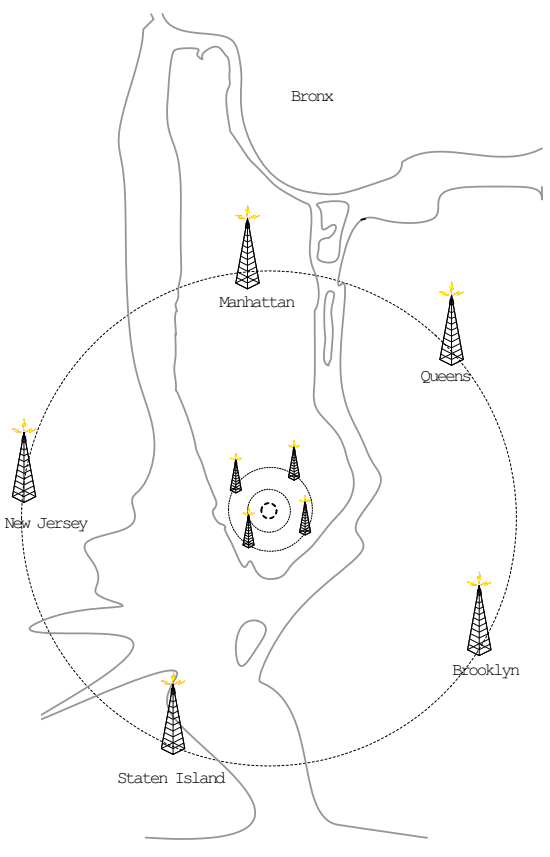

Figure 1: A Wireless Recovery Network for the 9/11 scenario. Antennas in the affected area pick up communication signals from mobile users or companies' uplinks and transmit them to the outside antennas to ensure the connectivity.

both by means of radio equipment or wireless LANs in ad hoc mode can be considered. Wireless LANs represent an inexpensive technology, that is widely adopted. The main drawback of deploying wireless LAN technology for network recovery purposes, is the limited throughput and the inherent unreliability. Both technologies were considered during our study and the model presented in this paper applies to both. It is interesting to note that wireless shadow networks can also be applied for quickly restoring the connectivity of cellular networks. Figure 2 shows a cellular wireless network, where the inner base-stations are destroyed and the wired connection between the base-stations in the affected area is no longer usable. One way to quickly restore the connectivity for the mobile users is by activating base-stations inside the affected area, that pick up user signals and direct these to the surrounding not affected base-stations, where the traffic is routed underground. The base-stations in the affected area can either be placed there after the disaster (e.g., on trucks) or the existing base-stations can be reconfigured for network recovery.

\subsection{Contribution}

Since activating too many wireless stations will cause interference and thereby significantly reduce the total system throughput, there is an optimum number of stations for a given geometry. Therefore, it makes sense to address the design problem of determining the desired number of wireless stations to obtain a certain throughput and study the influence of the location of the wireless nodes on the expected system throughput. In this paper, we present a model for analyzing wireless shadow networks and consider the design

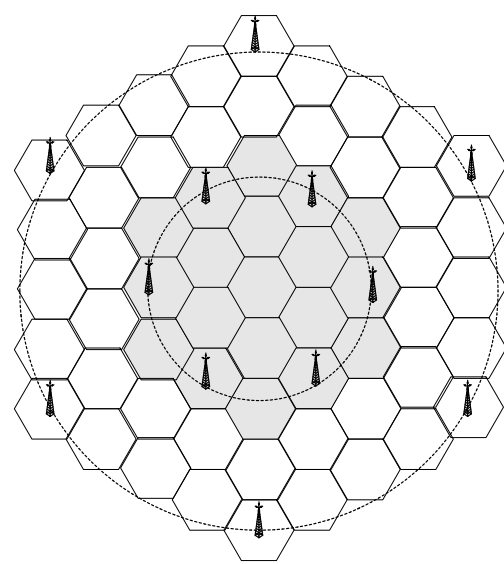

Figure 2: Recovery of a Cellular Network. The base stations in the marked cells are destroyed and the wired connection between the base stations is no longer usable. Restoration of the connectivity can be achieved by activating base stations in the affected area, that pick up the signals and direct them to the surrounding not affected base stations.

problem for a simple geometry. We derive analytical expressions for the expected wireless system throughput. The expressions allow us to obtain interesting rules of thumb for the design problem of where to locate the wireless nodes and how many are needed. The expected gain, when using directional antennas (as a function of beam-width), has been evaluated as well. Furthermore, some interesting applications and extensions of the model, which will be the subject of future publications, will be briefly introduced as well.

\subsection{Related Work}

Work on disaster recovery in the area of sensor networks mainly focuses on power control of the wireless nodes and routing. Diffusive routing in sensor networks is discussed in [3].

In [4], the authors present expressions for an upper and a lower bound for the transport capacity of a plane disk filled with wireless nodes. Both random and arbitrary network models are considered. Two interference models are analyzed (protocol model and physical model). In [5], these results are experimentally verified and some interesting throughput measurement are presented for chains and lattices of IEEE 802.11 [6] ad hoc nodes.

Communication performance evaluation results for ad hoc wireless networks are presented in [7]. Another interesting related area is loadbalancing and throughput maximization in ad hoc networks: the impact of alternative path routing for load balancing in mobile ad hoc networks is addressed in [8].

In order to increase the throughput of wireless systems, MIMO (Multiple Input Multiple Output [9]) systems, have been investigated. These systems consist of multiple antennas at both the transmitter and receiver sides, and rely on the assumption that the environment has plenty of multipath reflections).

This paper is structured as follows: section 2 presents the model assumptions and the considered geometric constella- 


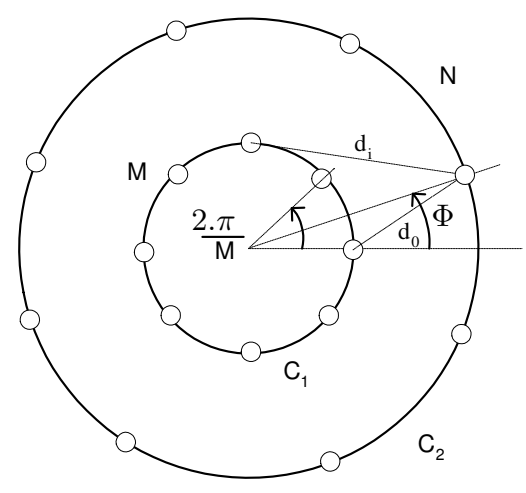

Figure 3: Simplified geometry: The inner circle $C_{1}$ contains the nodes inside the affected area, whereas the outer circle $C_{2}$ contains the network nodes, connected to a wired network. Wireless transmission takes place from the nodes on $C_{1}$ directly to nodes on $C_{2}$. In first instance, we will assume that nodes on $C_{1}$ and $C_{2}$ are equidistant. $\Phi$ denotes the angular distance between a node on $C_{1}$ and $C_{2}$.

tions. In section 3 , the analytical expressions for the throughput calculation of a wireless recovery network in equidistant constellation are detailed. In section 4, some interesting throughput results are shown, together with a description of the influence of the model parameters. In section 5, we consider the effect of using directional antennas. The model for taking directional antennas into account is presented and the influence of the beam-width on the wireless system throughput is investigated. In section 6 , we introduce the some interesting applications and extensions of the presented model. Finally, section 7 summarizes the paper and sums up the main conclusions, that can be drawn.

\section{MODEL DESCRIPTION}

\subsection{Assumptions}

(1) Circular Geometry: we assume that the nodes surrounding the affected area are located on a circle, as illustrated in Figure 3: on $C_{1}$ the antennas are located, that pick up the signals from the affected area and transmit them to the antennas on $C_{2}$. The nodes on $C_{2}$ route the received packets to their destination via a wired connection. Each node is characterized by its angle $\theta$ with a reference axis $(0 \leq \theta<2 . \pi)$. We assume that the antennas inside the affected area, that are either installed or activated, are located on a circle $C_{1}$ with radius $R_{1}$. $M$ denotes the number of nodes on $C_{1}$, whereas $N$ denotes the number of nodes on $C_{2}$. We refer to Figure 1 and 2 to motivate that this circular geometry assumption indeed makes sense.

(2) Radio Propagation Model: we assume a path-loss power law model. According to this model, the mean value received signal power $p$ (in Watts) is a decreasing function of the distance $d$ between transmitter and receiver and can be expressed as:

$$
p=c . d^{-\eta},
$$

where $c$ is the power constant of the transmitter (also defined as the power at distance zero), $\eta$ is the path loss exponent. Typical values for $\eta$ are listed in Table 1 [10]. The coverage area of a node is defined as a circle with radius $R_{\text {coverage }}$ around this node. A node can have direct communication with all nodes, that fall inside its coverage area. The received power outside the coverage area of a node is lower than a certain threshold value $\Gamma$ (A realistic value for $\Gamma$ in wireless LAN systems is $-82 \mathrm{dBm})$. Given c and $\eta, R_{\text {coverage }}$ follows from equation (1). For fixed $\eta$ and constant c values, $R_{\text {coverage }}$ can be considered as a model parameter. Given $R_{\text {coverage }}$ and $\eta$, a minimum value for $c$ can be calculated.

(3) Constant Path Loss Exponent: we assume that $\eta$ is constant for a given geometry and is not location or time dependent.

(4) Equal Transmitted Power and Activity Level: all nodes are assumed to transmit at the same power level. Nodes can not raise their power levels to obtain a better Signal to Interference (SIR) value at the receiver side. We also assume that the nodes have the same activity level. The activity level of a node is defined as the average amount of time that the node is sending or receiving packets.

(5) Spread Spectrum Techniques: in order to provide a reduction in the interference power and hence obtain a processing gain, spread spectrum techniques are often used. The processing gain is denoted by $g$. A typical value for $g$ is $10.4 \mathrm{~dB}$. Usage of Spread Spectrum techniques requires that each receiver is aware of the spreading code of the transmitter.

(6) Transmission Frequency: We assume that all transmission between $C_{1}$ and $C_{2}$ takes place in the same frequency band in one direction and another frequency band in the other direction. As an extension, we can investigate the case, where each transmitter can choose a frequency out of certain range of frequencies. As mentioned in the introduction, we assume that the communication from the wireless nodes to the antennas on $C_{1}$ also takes place in another frequency band to avoid interference with the communication between the nodes on $C_{1}$ and $C_{2}$ and vice versa.

(7) Receiver Signal Selection: each receiver on $C_{2}$ either (i) selects the strongest signal, i.e. the signal from the closest node on $C_{1}$ (closest in the distance-metric sense) or (ii) receives all signals with a SIR above threshold.

(8) MAC-related modes we distinguish between following two modes: (i) ST-Mode: Simultaneous Transmission, i.e., all nodes transmit independent from each other (no MAC protocol), (ii) IA-Mode: Interference Avoidance, i.e., there is a MAC protocol (e.g., CSMA/CA) to detect when the channel is busy, and there is no interference within the coverage area of nodes. 
Table 1: Typical values of $\eta$

\begin{tabular}{|c||c|}
\hline Environment & $\eta$ \\
\hline \hline Free space & 2 \\
\hline Urban Area & $2.7-3.5$ \\
\hline Shadowed Urban Area & $3-5$ \\
\hline In building & $4-6$ \\
\hline
\end{tabular}

(9) Interference: for correct reception of radio signals, the signal-to-interference ratio (SIR) needs to be higher than a certain threshold value. This threshold value is denoted by $\gamma$ and has for example a typical value of $7 \mathrm{~dB}$. SIR is defined as the ratio between the mean power of the wanted signal and the mean power of the sum of the interfering signals. In case the transmitters are equidistant, the SIR of a signal at a particular receiver can be shown to equal (taking the equal power and activity level assumption (3) into account):

$$
\begin{aligned}
\mathrm{SIR}= & \frac{\left(R_{1}^{2}+R_{2}^{2}-2 R_{1} R_{2} \cos \Phi\right)^{-\eta / 2}}{\sum_{i=1}^{M-1}\left(R_{1}^{2}+R_{2}^{2}-2 R_{1} R_{2} \cos \left(\frac{2 \pi i}{M}-\Phi\right)\right)^{-\eta / 2}} \\
& 0 \leq \Phi \leq \frac{\pi}{M},
\end{aligned}
$$

where $\Phi$ is the angular distance between the particular receiver and the transmitting node on $C_{1}$.

When making use of spreading techniques, the effective SIR can be expressed as:

$$
\mathrm{SIR}_{\mathrm{eff}}(\mathrm{dB})=\mathrm{SIR}(\mathrm{dB})+g(\mathrm{~dB})
$$

(10) Fading: In first instance, we neglect short term and long term fading variations around the signal's mean power. Rayleigh fading, Rician fading and Suzuki fading models can be taken into account at a later stage.

\subsection{Geometric Constellations}

We distinguish between 4 constellation scenarios:

(i) equidistant constellation, where the nodes on both circles are equidistant, (ii) perturbations on equidistant constellation, when node positions are not perfectly equidistant, we calculate the expected wireless system throughput as a function of the node location variations, (iii) entirely random constellation, when the node locations are uniform and independently distributed along the circles, and (iv) multihop constellations, which are constructed by superposition of different concentric circles.

\section{THROUGHPUT FOR EQUIDISTANT CONSTELLATION}

In this section, we assume that the nodes on both circles are equidistant, and that the relative phase between the two constellations is a random variable with a uniform distribution. We introduce the following notations:

- $n_{r}(\phi)$ : the number of receivers that can pick up the signal of one transmitter as a function of the angle $\phi$ of the transmitter with a reference axis.

- $n_{s}(\phi)$ : the number of different signals that are picked up by one receiver as a function of the angle $\phi$ of the receiver with a reference axis.
- $T_{s}(\phi)$ : the throughput of sent packets as a function of the relative phase between the equidistant constellations on $C_{1}$ and $C_{2}$.

- $T_{r}(\phi)$ : the throughput of received packets as a function of the relative phase between the equidistant constellations on $C_{1}$ and $C_{2}$.

- $T_{i}$ : the maximum throughput (in MBit/s) of a single active channel.

By their definition, following properties hold for $n_{r}(\phi)$, $n_{s}(\phi), T_{s}(\phi)$ and $T_{r}(\phi)$ :

$$
\begin{aligned}
n_{r}(\phi) & =n_{r}\left(\phi+\frac{2 \pi j}{N}\right) \forall j \text { integer } \\
n_{s}(\phi) & =n_{s}\left(\phi+\frac{2 \pi i}{M}\right) \forall i \text { integer } \\
T_{r}(\phi) & =\sum_{j=0}^{N-1} n_{s}\left(\phi+\frac{2 \pi j}{N}\right) \times T_{i} \\
T_{s}(\phi) & =\sum_{i=0}^{M-1}\left(n_{r}\left(\phi+\frac{2 \pi i}{M}\right)>0: 1: 0\right) \times T_{i},
\end{aligned}
$$

where (expr : $1: 0)$ yields 1 when expr is true and 0 otherwise. $T_{r}(\phi)$, the throughput of received packets is calculated by adding $N$ instances of the $n_{s}(\phi)$ curve, each shifted over $\frac{2 \pi}{N}$, whereas $T_{s}(\phi)$, the throughput of sent packets is calculated by adding $M$ instances of the "binary version" of the $n_{r}(\phi)$ curve, each shifted over $\frac{2 \pi}{M}$.

\section{1 $n_{r}(\phi)$ calculation}

Figure 4 shows the $\operatorname{SIR}_{r}(\phi)$ for a signal from a transmitter experienced by a receiver at an angular distance of $\phi$.

$$
\operatorname{SIR}_{r}(\phi)=\operatorname{SIR}_{r}\left(\phi+\frac{2 \pi j}{N}\right) \forall j \text { integer }
$$

The intersection-point with $\mathrm{SIR}_{r}=\gamma$ line gives the maximum angular distance between the particular transmitter and a receiver to be picked up by that receiver. Let's call this maximum phase $\phi_{r}^{\max } \cdot n_{r}(\phi)$ is then simply the number of receivers that are in the interval: $\left[\phi-\phi_{r}^{\max }, \phi+\phi_{r}^{\max }\right]$. The larger this $\phi_{r}^{\max }$, the broader the peaks in the $n_{r}(\phi)$ curve. Note that $\operatorname{SIR}_{r}(\phi)$ is independent of $N$, but dependent on $M$.

\section{2 $n_{s}(\phi)$ calculation}

Due to symmetry reasons, Figure 4 also shows the $\operatorname{SIR}_{s}(\phi)$ at a receiver for a signal from a transmitter at an angular distance of $\phi$.

$$
\phi_{s}^{\max }=\phi_{r}^{\max }=\phi^{\max }
$$

$\phi^{\max }$ determines the whole system behavior and is a function of $\frac{R_{2}}{R_{1}}, M, \eta$ and $\gamma$ :

$$
\phi^{\max }=\phi^{\max }\left(\frac{R_{2}}{R_{1}}, M, \eta, \gamma\right)
$$

In summary, $\phi^{\max }$ can be defined as the implicit aperture of a transmitter or receiver in the system (measured from the center of the circles): (i) for a transmitter, it defines the angle, within which all receivers can pickup the signal from this transmitter and (ii) for a receiver, it defines the angle 


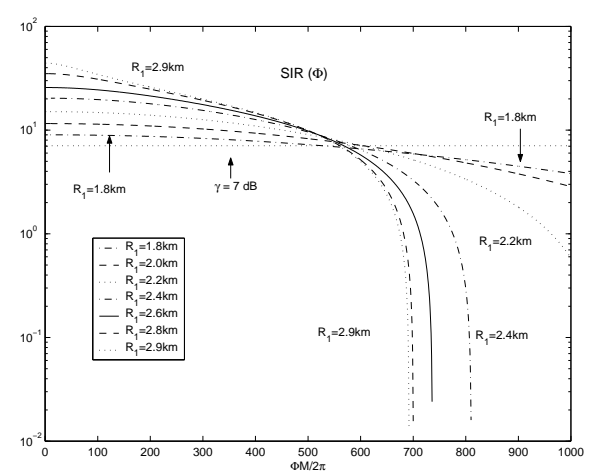

Figure 4: $\operatorname{SIR}_{r}(\phi)$ for a signal from a particular transmitter experienced by a receiver at an angular distance of $\phi(M=10)$

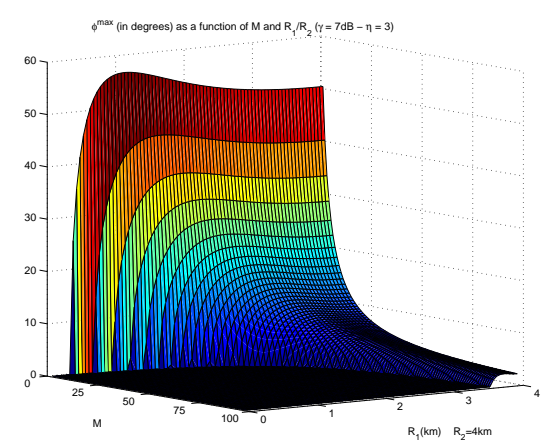

Figure 5: $\phi^{\max }$ as a function of $\frac{R_{1}}{R_{2}}$ and $M(\gamma=\mathbf{7 d B}$, $g=10.4 \mathrm{~dB}, \eta=3$ )

within which the signal from all transmitters can be picked up.

$\phi^{\max }$ is shown in Figure 5 as a function of $M$ and $\frac{R_{1}}{R_{2}}$.

\subsection{Expected Throughput Calculation}

Assuming that the phase between the two equidistant constellations on the circles is uniformly distributed, following expressions hold for $n_{r}$ and $n_{s}$ :

$$
\begin{aligned}
\left\lfloor\frac{N \phi^{\max }}{\pi}\right\rfloor \leq & n_{r} \leq\left\lceil\frac{N \phi^{\max }}{\pi}\right\rceil \\
E\left[n_{r}\right] & =\frac{N \phi^{\max }}{\pi} \\
\left\lfloor\frac{M \phi^{\max }}{\pi}\right\rfloor \leq & n_{s} \leq\left\lceil\frac{M \phi^{\max }}{\pi}\right\rceil \\
E\left[n_{s}\right] & =\frac{M \phi^{\max }}{\pi} \\
\frac{E\left[n_{s}\right]}{E\left[n_{r}\right]} & =\frac{M}{N}
\end{aligned}
$$

where $\lfloor x\rfloor$ denotes the largest integer, smaller than or equal to $x,\lceil x\rceil$ denotes the smallest integer, greater than or equal to $x$ and $\mathrm{E}[$.$] denotes the expected value of the$ stochastic variable between brackets. We can also calculate the expected values for $T_{r}(\phi)$ and $T_{s}(\phi)$ :

$$
\begin{aligned}
& E\left[T_{r}\right]=\frac{M N \phi^{\max }}{\pi} \\
& E\left[T_{s}\right]=\left\{\begin{array}{cc}
M & \frac{N \phi^{\max }}{N \phi^{\text {max }}} \geq 1 \\
\frac{M N \phi^{\max }}{\pi} & \frac{N}{\pi}
\end{array}\right. \\
& \frac{E\left[T_{r}\right]}{E\left[T_{s}\right]}=\left\{\begin{array}{cc}
\frac{N \phi^{\max }}{\pi} & \frac{N \phi^{\max }}{\pi^{m a x}} \geq 1 \\
1 & \frac{N \phi^{\text {max }}}{\pi}<1
\end{array}\right.
\end{aligned}
$$

Note that the expected throughput in the other direction (i.e., from $C_{2}$ to $C_{1}$ ) can be calculated likewise. Equation (23) implies that $E\left[T_{r}\right] \geq E\left[T_{s}\right]$ : the expected throughput of received packets is always higher than or equal to the expected throughput of sent packets. This is due to the fact that a transmitted signal can be picked up by multiple receivers (duplication of packets) and for the $E\left[T_{s}\right]$ calculation, those duplicated packets are not counted.

\section{THROUGHPUT EVALUATION}

\subsection{Optimal Number of Transmitters}

Figure 6(a) shows the expected throughput as a function of $\mathrm{M}$, the number of transmitter nodes for different values of $R_{2}-R_{1}$ and $\mathrm{N}=10$. The throughput heavily depends on the distance between the two circles: the further away from a receiver, the faster the expected throughput drops when more transmitters are activated. This effect is due to the smaller distance differences, when the radius of the inner circle decreases. It is interesting to note that, for small $R_{2}-R_{1}$, the expected throughput very slowly increases when activating more transmitters and much more transmitters are required to obtain the same expected throughput as in the case of larger $R_{2}-R_{1}$. For given $N$, there is a clearly a preferred value for $R_{1} / R_{2}$ and $M$. From our model, it follows that the best choice is $R_{1} / R_{2}=0.8$ and $N \leq M \leq 2 N$. For this value, the expected throughput curve obtains its maximum for a reasonable amount of activated transmitter nodes and around the maximum, the expected throughput doesn't drop suddenly when an extra transmitter is activated.

Figure 6(b) shows the expected throughput for different values of $\mathrm{N}$, the number of available receivers and $R_{2}-R_{1}$. It follows that the larger $\mathrm{N}$, the faster the decrease of the expected throughput when more transmitters are activated. The larger $N$, the more sensitive the choice of $M$ becomes and the more sudden the expected throughput drops when more transmitter nodes are activated.

Note that $E\left[T_{r}\right]$ can be easily derived from $E\left[T_{s}\right]$, by using equation (18).

\subsection{Influence of $\eta$}

Figures 7(a), 7(b), 7(c) show the expected system throughput as a function of $\mathrm{M}$ and $\eta$ for $R_{2}=3 \mathrm{~km}$ and $R_{1}=2.8 \mathrm{~km}$, $R_{1}=2 \mathrm{~km}, R_{1}=0.5 \mathrm{~km}$, respectively. We observe the following behavior: the larger $\eta$, the less interference from the surrounding nodes and the higher the expected throughput can grow. For small values of $R_{2}-R_{1}$ and large values of $\eta$, we observe that the expected throughput very slowly increases when activating more transmitters. In this case, more transmitters are required to obtain the same expected throughput as in the case of smaller $\eta$. This is due to the fact that for smaller $\eta$, a receiver can pick up signals from 


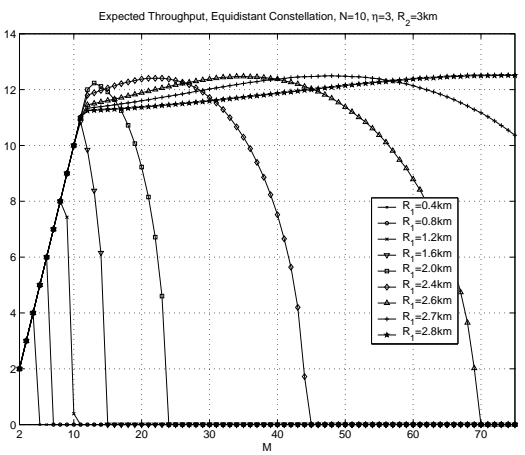

(a)

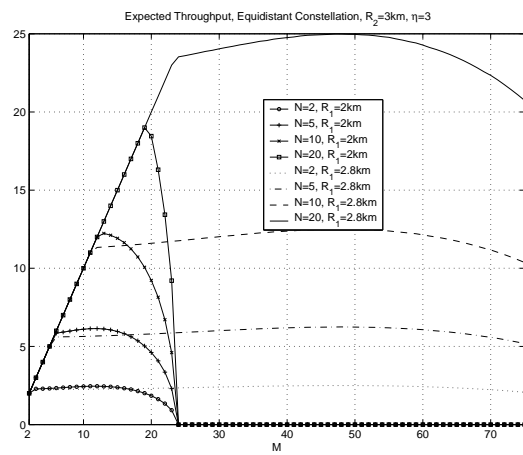

(b)

Figure 6: Expected throughput $E\left[T_{s}\right]$ as a function of $\mathbf{M}$ (=the number of activated transmitters), $R_{2}=3 \mathrm{~km}$ : (a) for different values of $R_{2}-R_{1}$, (b) for $N=\mathbf{2 , 5 , 1 0 , 2 0}$ and $R_{1}=\mathbf{2} \mathrm{km}$ and $2.8 \mathrm{~km}(\gamma=\mathbf{7 B}, g=10.4 \mathrm{~dB}, \eta=3)$.

multiple nodes (thanks to the use of spread spectrum techniques) for a small number of transmitters (i.e., for larger distance between that rceiver and the transmitters, due to the equidistance assumption). For larger $\eta$, the receivers are only able to pick up signals from multiple receivers when they are close enough.

Note that, since large $\eta$-values imply a small coverage area, this evaluation only makes good sense for small values of $R_{2}-R_{1}$.

\section{DIRECTIONAL ANTENNAS}

In this section, we examine the influence of the transmitter beam-width $\zeta$. As shown in Figure 8, the beam-width is defined as the angle $\zeta$, originating from a transmitter: only nodes inside the defined segment can receive the transmitted signal. We also assume that (i) the power received by a node inside the angle $\zeta$ is independent of the node's angular distance to the transmitter, (ii) the antennas are directed orthogonal to $C_{1}$ and (iii) the phase between the two equidistant constellations on the circles is uniformly distributed.

\subsection{SIR Calculation}

In the equidistant case, the Signal to Interference Ratio (SIR) can be calculated as:

$$
\begin{gathered}
S I R=\frac{\left(R_{1}^{2}+R_{2}^{2}-2 R_{1} R_{2} \cdot \cos \Phi\right)^{-\eta / 2}}{2 \cdot \sum_{i=1}^{i} \max _{1}^{2}\left(R_{1}^{2}+R_{2}^{2}-2 R_{1} R_{2} \cdot \cos \left(\frac{2 \pi i}{M}+\Phi\right)\right)^{-\eta / 2}} \\
0 \leq \Phi \leq \frac{\pi}{M}
\end{gathered}
$$

where $i_{\max }$ is the highest index of the nodes that transmit power to the receiver under consideration. Given $\zeta, \Phi, M$, $R_{1}$ and $R_{2}, i_{\max }$ can be easily determined. We consider the triangle of figure 8. In order for node $i$ on $C_{1}$ to be received at a particular node on $C_{2}$, the following two inequalities have to be satisfied:

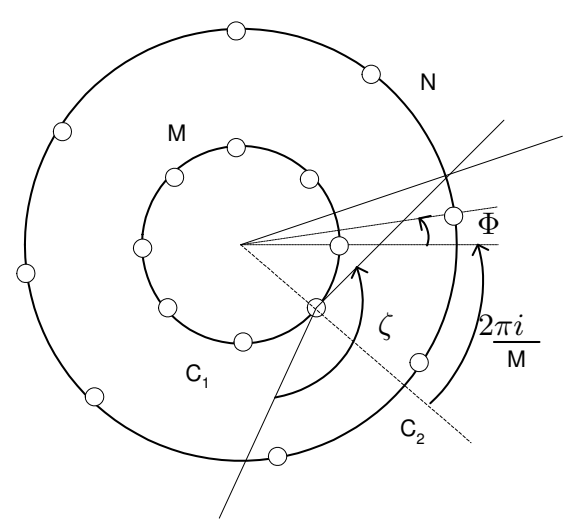

Figure 8: Equidistant constellation with phase $\Phi$ and beam-width $\zeta$.

$$
\begin{array}{r}
\frac{\zeta}{2}-\Phi-\frac{2 . \pi \cdot i}{M}>0 \\
\frac{R_{1} \sin \left(\pi-\frac{\zeta}{2}\right)}{\sin \left(\frac{\zeta}{2}-\Phi-\frac{2 \pi i}{M}\right)}<R_{2}
\end{array}
$$

From (21), $i_{\max }$ follows:

$$
i_{\text {max }}=\left\lfloor M \frac{\frac{\zeta}{2}-\Phi-\arcsin \left(\frac{R_{1} \sin \left(\pi-\frac{\zeta}{2}\right)}{R_{2}}\right)}{2 \pi}\right\rfloor
$$

We can determine $\phi_{\max }\left(\zeta, M, R_{1} / R_{2}, \eta, \gamma\right)$ and apply the expected throughput calculation method, detailed in section III. We expect an improvement of the performance, since less terms (all strictly positive) are to be taken into account in the denominator.

\subsection{Determination of $\phi^{\max }(\zeta)$}

$\phi^{\max }(\zeta)$ can be determined by finding the value of $\phi$, which yields a SIR of $\gamma \mathrm{dB}$ (receiver sensitivity). When changing the $\phi$ value, transmitters can get out of range for a receiver 


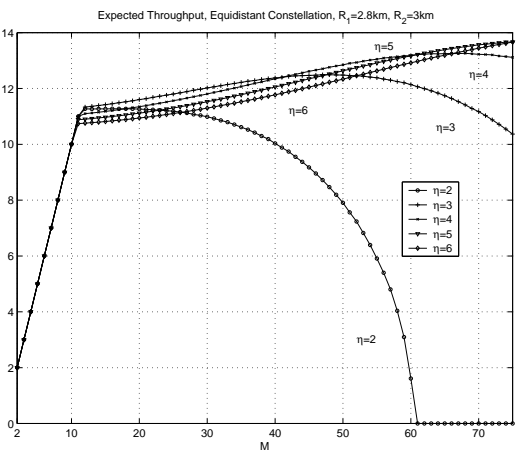

(a) $\mathrm{R} 1=2.8 \mathrm{~km}$

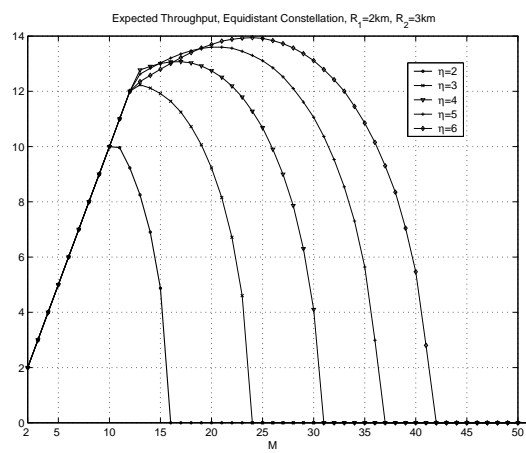

(b) $\mathrm{R} 1=2 \mathrm{~km}$

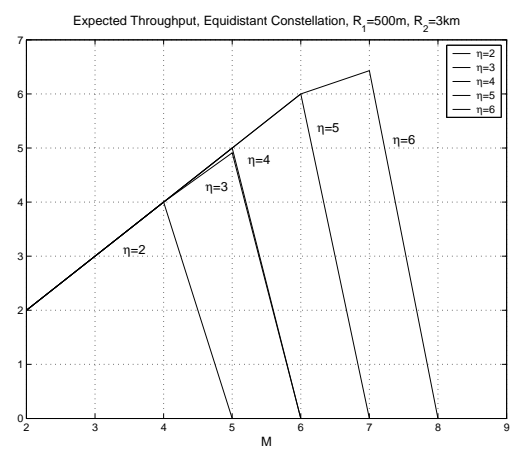

(c) $\mathrm{R} 1=0.5 \mathrm{~km}$

Figure 7: Expected throughput $E\left[T_{s}\right]$ as a function of $\mathbf{M}$ (=the number of activated transmitters for different values of $\eta\left(R_{2}=3 \mathrm{~km}, \mathbf{N}=\mathbf{1 0}, \gamma=\mathbf{7 d B}, g=10.4 \mathrm{~dB}\right)$.

or similarly some transmitters can get in range and start interfering with a communication channel. Therefore, $\operatorname{SIR}(\phi)$ is not a monotonous function and $\phi^{\max }(\zeta)$ can be the sum of interval lengths, where $\operatorname{SIR}(\phi) \geq \gamma$. We implemented a mathematical tool to determine $\phi^{\max }(\zeta)$ for given $\mathrm{M}, R_{1} / R_{2}$, $\eta, \gamma$ and $g$. In the subsection below, some evaluation results are presented.

\subsection{Evaluation Results}

In Figure 9, the expected throughput is shown for three values of $R_{1}\left(R_{2}=3 \mathrm{~km}\right)$ and for different values of $\zeta$, as a function of $\mathrm{M}$ (the number of activated transmitters). The smaller $\zeta$, the slower the throughput grows when additional transmitters are activated. However, for small $\zeta$, the throughput obtains higher values. The initial slope of the throughput curve is dependent on $\zeta$ : the smaller $\zeta$, the higher the probability that a receiver is out of scope for a transmitter (due to the fact that $\Phi$ is assumed to be a uniformly distributed stochastic variable). When the number of activated transmitter nodes increases, more transmitter nodes will be picked up by a receiver node and more will be picked up when the transmission angle $\zeta$ gets smaller. The optimal $\zeta$ clearly depends on $R_{1} / R_{2}$ : for small $R_{1} / R_{2}$ a small $\zeta$ is preferred, for larger $R_{1} / R_{2}$ we are better off with a large $\zeta$, to reduce the required amount of activated transmitter nodes. From the plots, shown in Figure 9, we are able to determine the optimal $\zeta$ and $M$, for given $R_{1} / R_{2}$ and $N$.

\section{MODEL APPLICATIONS AND EXTENSIONS}

Following interesting applications and extensions of the presented model can be thought of (these are the subject of current research and will be published later):

(1) Influence of small perturbations of the nodes' location on the system throughput: we assume that each transmitter node is uniformly distributed in the interval $\left(\phi-\frac{\theta_{1}}{2}, \phi+\frac{\theta_{1}}{2}\right)$, where $\phi$ represents the location of the node in the perfect equidistant case. $\theta_{1}$ represents the angular interval of each transmitter node. Similarly, we assume that each receiver node is uniformly distributed in the interval $\left(\phi-\frac{\theta_{2}}{2}, \phi+\frac{\theta_{2}}{2}\right)$, where $\theta_{2}$ represents the angular interval of each receiver node. In this case, we can no longer apply the easy $\phi^{\max }$ determination method explained above. Since $\phi^{\max }$ now is a stochastic parameter, we have to determine its probability density function. This probability density function is obtained by applying the following relationship between $\phi^{\max }$ and $\operatorname{SIR}(\phi)$ :

$$
\operatorname{Prob}\left[\phi^{\max }<\phi\right]=\operatorname{Prob}[\operatorname{SIR}(\phi)>\gamma]
$$

From this probability density function, the expected value of $\phi^{\text {max }}$ can be obtained. This expected value is denoted by $E\left[\phi^{\max }\right]$.

In case the receiver nodes are equidistant along circle $C_{2}$, the expected values of $n_{r}$ and $n_{s}$ can be calculated as:

$$
\begin{aligned}
E\left[n_{r}\right] & =\frac{N \times E\left[\phi^{\max }\right]}{\pi} \\
E\left[n_{s}\right] & =\frac{M \times E\left[\phi^{\max }\right]}{\pi} \\
\frac{E\left[n_{s}\right]}{E\left[n_{r}\right]} & =\frac{M}{N}
\end{aligned}
$$

and $E\left[T_{r}\right]$ and $E\left[T_{s}\right]$ can also be determined, similarly as in section III. For random receiver constellations the expected throughput can be calculated as well.

(2) Evaluation of multi-hop constellations: multi-hop constellations make use of relay nodes to achieve a larger geographic coverage. We assume that the relay nodes are on concentric circles between $C_{1}$ and $C_{2}$. When we assume that the constellations do not interfere, the total system throughput can be calculated as the minimum throughput over all involved circles. We have derived analytical expressions to determine the required number of hops to obtain a desired throughput $T^{*}$ as a function of the radii of the inner and outer circles, and the other system parameters.

(3) Capacity addition problem: this topic addresses the issue of how best to add capacity when the nodes are in 


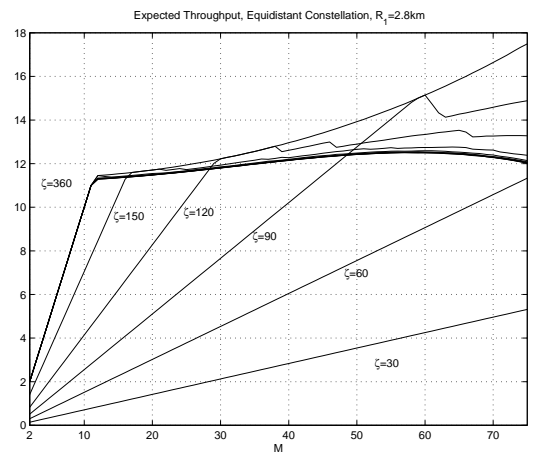

(a) $\mathrm{R} 1=2.8 \mathrm{~km}$

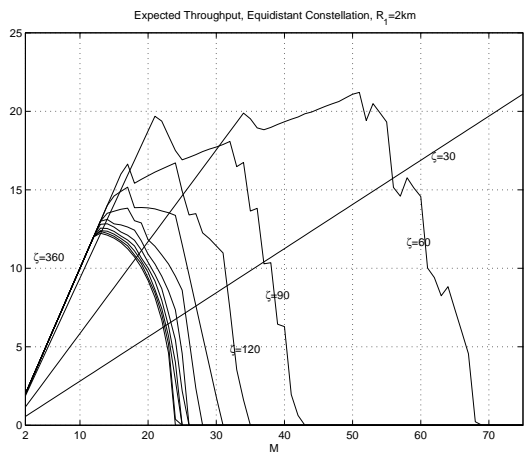

(b) $\mathrm{R} 1=2 \mathrm{~km}$

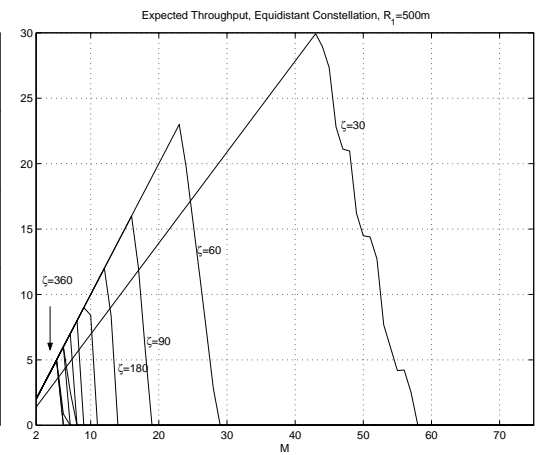

(c) $\mathrm{R} 1=0.5 \mathrm{~km}$

Figure 9: Expected throughput $E\left[T_{s}\right]$ as a function of $\mathbf{M}$ (=the number of activated transmitters for different values of $\zeta(30,60,90,120,150,180,210,240,270,300,330,360$ degrees $)\left(R_{2}=3 \mathrm{~km}, \mathbf{N}=10\right)$.

a given constellation and extra throughput is required, i.e. the determination of how many nodes should be added and whether the existing nodes be moved to another location in order to obtain a higher throughput.

(4) Antenna steering game: we consider a game, where the antennas can be directed towards a receiver with the goal of increasing the SIR of a signal at a particular receiver. In case the antennas can be steered towards a receiver, we expected the throughput to increase. Several antenna steering strategies can be compared in terms of expected throughput and the expected gain of a cooperative strategy versus a non-cooperative strategy are being studied at the moment of writing.

\section{CONCLUSION}

In this paper, wireless shadow networks were introduced for recovery of network services in case of catastrophic failures. We motivated the importance of modeling wireless shadow networks and presented a model for analyzing their behavior as a function of the system parameters. For the equidistant constellation, we showed that the system behavior can be described by a single parameter $\phi_{\max }$, that depends on the activated number of transmitters, the distance between the transmitters and the receivers, the path loss exponent $\eta$ and the receiver sensitivity $\gamma$.

Some interesting rules of thumb of where to locate the nodes and how many, have been derived. The expected gain resulting from using directional antennas (as a function of beam-width) has been also investigated. Finally, our model calls for some extensions: (i) the modeling of constellations, which are not perfectly equidistant or where the nodes are not perfectly located on circles, (ii) the modeling of multi-hop constellations and their influence on the expected throughput, (iii) the capacity addition problem, i.e. study strategies for adding capacity to a given constellation of wireless nodes and (iv) the study and the comparison of the cooperative versus non-cooperative behavior of steering the directional antennas to a specific receiver. The results of these extensions will be the subject of a later publication.

\section{REFERENCES}

[1] P. Demeester, T. Wu, and N. Yoshikai, "Survivable communication networks," IEEE Communications Magazine, Special Issue on Survivable Communications Networks, vol. 37, no. 8, August 1999.

[2] B. V. Caenegem, W. V. Parys, F. D. Turck, and P. M. Demeester, "Dimensioning of survivable wdm networks," IEEE JSAC, vol. 15, no. 7, September 1998.

[3] C. Intanagonwiwat, R. Govindan, and D. Estrin, "Directed diffusion: A scalable and robust communication paradigm for sensor networks," in Proceedings of the Sixth Annual International Conference on Mobile Computing and Networking (MobiCOM '00), Boston, Massachussetts, 2000.

[4] P. Gupta and P. Kumar, "The capacity of wireless networks," IEEE Transactions on Information Theory, vol. 46, no. 2, pp. 388-404, 2000.

[5] J. Li, C. Blake, D. D. Couto, H. I. Lee, and R. Morris, "Capacity of ad hoc wireless networks," in Proc. IEEEMOBICOM 2003, Rome, Italy, 2001.

[6] Wireless LAN Medium Access Control (MAC) and Physical Layer (PHY) Specifications, IEEE Computer Society LAN MAN Standards Committee IEEE Std. 802.11-1997, 1997.

[7] C.-K. Toh, M. Delwar, and D. Allen, "Evaluating the communication performance of an ad hoc wireless network," IEEE Transactions on Wireless Communications, vol. 1, no. 3, pp. 402-414, 2002.

[8] M. Pearlman, Z. Haas, P. Sholander, and S. Tabrizi, "the impact of alternate path routing for load balancing in mobile ad hoc networks," in Proceedings of the ACM MobiHoc, 2000, pp. 3-10.

[9] M. Shafi, D. Gesbert, S. Da-shan, P. Smith, and W. Tranter, "Mimo systems and applications," Selected Areas in Communications, IEEE Journal on, vol. 21, no. 5, June 2003.

[10] T. Rappaport, Wireless Communications, Principles and Practice. Prentice-Hall PTR, 2002. 